\title{
Stochastic RUL Calculation Enhanced With TDNN-Based IGBT Failure Modeling
}

\author{
Alireza Alghassi, Mohammad Samie, and Suresh Perinpanayagam
}

\begin{abstract}
Power electronics are widely used in the transport and energy sectors. Hence, the reliability of these power electronic components is critical to reducing the maintenance cost of these assets. It is vital that the health of these components is monitored for increasing the safety and availability of a system. The aim of this paper is to develop a prognostic technique for estimating the remaining useful life (RUL) of power electronic components. There is a need for an efficient prognostic algorithm that is embeddable and able to support on-board real-time decision-making. A time delay neural network (TDNN) is used in the development of failure modes for an insulated gate bipolar transistor (IGBT). Initially, the time delay neural network is constructed from training IGBTs' ageing samples. A stochastic process is performed for the estimation results to compute the probability of the health state during the degradation process. The proposed TDNN fusion with a statistical approach benefits the probability distribution function by improving the accuracy of the results of the TDDN in RUL prediction. The RUL (i.e., mean and confidence bounds) is then calculated from the simulation of the estimated degradation states. The prognostic results are evaluated using root mean square error (RMSE) and relative accuracy $(\mathrm{RA})$ prognostic evaluation metrics.
\end{abstract}

Index Terms-Insulated gate bipolar transistor (IGBT), power electronics, prognostics, probability distribution function, remaining useful life (RUL), time-delay neural network.

\section{INTRODUCTION}

A $\mathrm{N}$ insulated gate bipolar transistor (IGBT) that is a voltage-controlled bipolar device used in switching applications is one of the most challenging power electronics components in terms of reliability. Improving efficiency, reliability and life time of such a device require intensive efforts on developing diagnostics and prognostics models. Prognostic that is technique to predict feature state of system which is predominantly used for estimating of life cycle usage of the device in real time operating. This is usually known as remaining useful life (RUL) in the field of asset management. In this context, reliability of power electronics plays an increasingly critical role in the energy and transport sectors. For example, IGBTs uses in Railway Applications are considered to be leading edge critical device whose failure rate increases the

Manuscript received November 12, 2014; revised January 22, 2015, June 29, 2015, October 13, 2015; accepted October 15, 2015. Associate Editor: E. Zio.

The authors are with the School of Aerospace, Transport and Manufacturing, Cranfield University, Bedford MK43 0A1 U.K.

Color versions of one or more of the figures in this paper are available online at $\mathrm{http}: / /$ ieeexplore.ieee.org.

Digital Object Identifier 10.1109/TR.2015.2499960 overall probability of malfunction in railway traction chains. In this regard, prognostic evaluation of IGBT can be extremely useful for train-line maintenance crews who need to assess which modules can be deferred for repair and which must be replaced within the maintenance window. Once prognostic results are used to address and predict the particular failure mode, the component whose repair cannot be deferred can be classified in term of severity and the availability of the parts can be scheduled, thereby avoiding delay due to unscheduled maintenance.

Therefore, the reliability of power electronics is a vital issue for the commercial success of industrial applications. Initially, in such applications, IGBT module life cycle expectancy is estimated at several thousands of hours. However, their lifetime under excessive temperatures or in other harsh environments will be much shorter, also depending on application, i.e., pulsed power. As a solution, diagnostic and prognostic approaches are the two principal aspects of condition-based maintenance (CBM) that are developed to increase the useful life time of IGBTs by monitoring critical parameters such as $V_{C E}, I_{C E}$ [1].

As systems mainly work in critical harsh and noisy environments, sensory data is highly polluted with noise. Hence, robust prognostic models that mitigate uncertainty are necessary However, a wide range of numerical and statistical methods as well as well-known machine learning approaches have been employed for the development of prognostic models. The imponderables of the associated uncertainties surrounding future health estimation have not been taken into account in which have been introduced by incomplete failure model. The aim of this paper is to improve the RUL estimation by fusing a probabilistic function with a TDNN failure model which uses less computational power and in comparison to all neural networks is very fast. Furthermore, it progressively allows uncertainty bounds indicate true value, thus it provides benefit of confidence bounds for the decision makers [2].

This paper bases its study on a failure data sets derived from a power cycling test rig wherein IGBTs were exposed to cyclic temperatures similar to those encountered in railway traction applications. We introduce an approach for the development of a time delay neural network for failure modelling using health state classification fused with probabilistic Monte Carlo RUL simulation, thus enabling us a significant reduction of the uncertainty occurring from an incomplete failure model and enhancing the clear indication of the current health state as well as improvement the RUL calculation. This has been successfully employed in the accurate and robust prediction of the current degradation of the device and allows better decisions to be made 


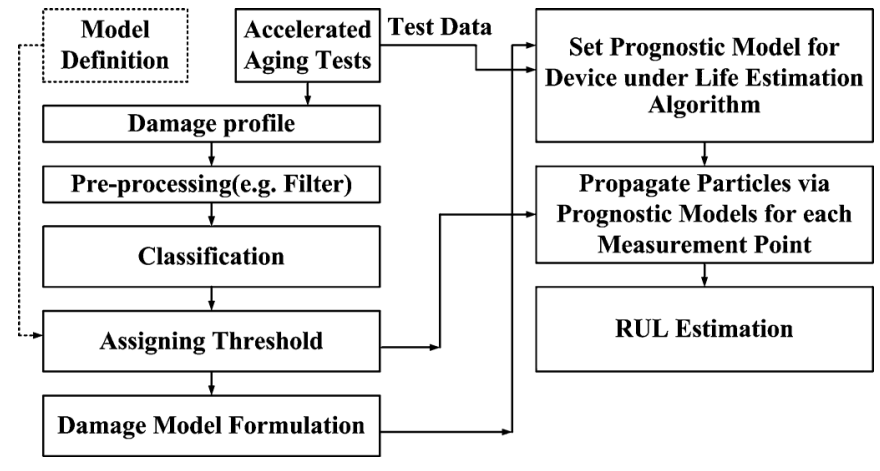

Fig. 1. Algorithm to develop prognostics model.

regarding scheduling of maintenance policies in integrated vehicle health management (IVHM).

This paper is organized as follows. Section II summarizes the steps in the prognostic approach, including data-driven and hybrid models. The next section describes ageing experiments and summarizes failure mechanisms. It also discusses the accelerated ageing test setup and the degradation profile of IGBTs. Section IV discuses degradation models, problem formulation and the time-delay neural network (TDNN) approach. Section V discusses the prognostic approach using the maximum likelihood method for IGBT model optimization. It also discusses the Monte Carlo simulation for calculating the area under the curve of the estimated IGBT degradation model and presents the proposed RUL algorithm. Section VI discusses the prognostic results using root mean square error (RMSE). Concluding remarks and future work are discussed in Section VII.

\section{Conventional Prognostics Models}

The current prognostic approaches for power electronics can be classified into three methods: 1) a data-driven approach; 2) the physics-of-failure (POF); and 3) fusion methods or hybrid models which integrate the data-driven and POF approaches. Fig. 1 summarizes the basic knowledge needed to develop data driven based and model-based prognostics. As shown in this figure, the development of prognostic models is generally started with the definition of a model for model-based prognostics that requires understanding the physics-of-failure of the IGBT component. It is often a difficult task to establish an accurate physical model. On the other hand, data-driven approaches start with accelerating aging tests followed with pre-processing (filtering) and classification for eliminating noise from collected data, and damage model formulation followed by RUL estimation. There is also an important initial step (known as feature extraction step) to investigate what signals should be monitored that would allow prognostics to be successfully developed based on features extracted from those signals [3], [4].

The collection of data requires accelerated ageing tests which are used to fail devices parts at high temperatures in a shorter time frame under various working conditions such as high baseplate temperature $\left(60^{\circ} \mathrm{C}\right.$ to $\left.100^{\circ} \mathrm{C}\right)$ and large temperature swings $\left(60{ }^{\circ} \mathrm{C}\right.$ to $\left.120{ }^{\circ} \mathrm{C}\right)$, these values are depending on system applications. In an accelerated ageing test, the particular wear-out mechanisms which are of interest accelerate to cause failure. The part/device is monitored throughout the test, noting the critical parameters for the particular device. Accelerated ageing tests which are used to obtain a detailed understanding of IGBT failure modes, and in turn, determine parameters are indicative of the various failure mechanisms. Employing these precursor parameters are prerequisite to develop a forecasting model for observing significant changes in the current health of the device and predicted failure [5]. However, precursor parameter identification for power electronics prognostics is challenging due to the representation of the uncertainties in the degradation profile for the precursor parameter. The precursor also uses to monitor direct or indirect failure mechanisms trend in order enables the prognostic model estimates the longest possible RUL to facilitate the maintenance of the power electronics. Hence, an advanced approach to the development of a prognostic tool for IGBTs is required. This could then be used to forecast failures, improve system life, and reduce unnecessary maintenance [6].

Following the identification of parameters which are a precursor to failure, the feature extraction (i.e., mean, median) and clustering of the collected data (i.e., finding the number of states for the degradation profile) are carried out to provide information for training a prognostic model. The trained model is given a predefined threshold value which is used in the prognostic algorithm. Data-driven methods are subjected to two different approaches. The first approach is known as a direct estimation of RUL in which the model learns directly from the damage data set. In contrast, the second indirect approach initially needs to estimate a damage progression model and then propagates the expected data through the model until a predefined threshold for the RUL is reached. The difficulty with the second method is that obtaining a reliable failure data set is often challenging due to various between devices and complete knowledge of the component is often unavailable [7].

Following the classification/clustering step, a damage model is created using data-mining techniques that ultimately model the failure mechanisms of the IGBT. Although there are a number of popular conventional numerical methods (such as Kalman filters, particle filters, machine-learning approaches, and support vector machines [8]) used for developing degradation models in a wide range of different applications, only a few of them have been employed so far for the modelling of degradation of IGBTs. A Kalman filter (KF) tunes and updates the parameters of the model on stream noisy input test data for linear systems. In contrast, a particle filter updates the parameters of the model with the most weighted samples generated from probability distribution of the system which mitigates the degeneracy of particles by a number of iterations.

In general, developing versatile physical model for both Kalman and particle filter is necessary to present a degradation model that avoids the main disadvantage of both techniques. The KF can be a good estimator if it is given a precise state-space model which constructs from all interpretable unobservable dynamic parameters of component. Overall, imprecise knowledge of the model parameter and inaccurate initialization of the filter lead to inconsistence with true component failure model [9]. Our proposed prognostics approach overcomes the 
complexity of developing the PoF, learns the degradation pattern online, and adapts itself with the dynamic of the parameter trend which will create a robust failure model.

In [10], Patil et al. proposed early anomaly detection implementing Mahalanobis distance (MD) using on-state collector-emitter voltage $\left(V_{c e}\right)$ and collector-emitter current (Ice) as precursor parameters and a particle filter approach to calculate RUL before catastrophic failure occurs. Patil et al. present an anomaly detection parameter which is used as a diagnostic parameter. However, in term of prognostic technique, they have introduced the mean time to failure estimation relatively has considerable divergent from parametric failure. The RUL evaluation metric also just carried out from anomaly detection till the failure threshold thus, the result will expect highly greater than $21 \%$ prediction error and less precision.

Celaya et al. develop a prognostic algorithm technique using an extended KF for MOSFET component [11]. The algorithm claims to be a versatile candidate for component level RUL calculation. The on-state resistance of the device is chosen as a precursor parameter which has increased due to die attached degradation process. The accuracy of the prognostics results has been challenged using evaluation metric relative error (RA).

In [12], Saha et al. implement a particle filter to calculate RUL and Monte Carlo (MC) method for simulation results. The reliable run-to-failure data set was obtained by conducting thermal overstress accelerated ageing tests on a IRG4BC30KD IGBT. Third-order polynomial fit of the tail of the Ice waveform was used to create a failure precursor model. In [12] data manipulation and model base learning using regression analyses were performed while offline. Furthermore, the particle filter has implemented on a model based approach for just the tail of the collector emitter current as a precursor parameter which almost IGBT approaches to catastrophic failure. Saha's model has not been developed for the entire degradation process and the strategy of failure model learning close to critical condition will not be applicable for maintenance decision makers.

In [13], Musallam et al. propose a real-time rain flow algorithm to calculate the full thermal cycle of the load profile (thermal profile) obtained from an IGBT electro-thermal model. The Coffin-Manson model is then utilized to estimate the endof-life of the IGBT bond wire interconnects and the substratesolder joints.

The Center for Advance Life Cycle Engineering (CALCE) proposes a hybrid prognostic method which fuses the data-driven and physics-of-failure approaches (PoF) to calculate RUL [14]. The PoF contains the systems components failure mechanisms, data base and data driven analyses, and the degradation level of the system to feed the PoF model for estimation of the future health state of the system.

\section{AgEING EXPERIMENT}

A review of IGBT failures and wear-out mechanisms is beneficial for this research. This information could then be used to identify precursor parameters that are indicative of prevalent failures. Subsequently, they will be used in the development of the diagnostic and prognostic algorithm to predict failure. This section provides details of the failure mode mechanisms and effects of power electronics, specifically IGBTs, based on the life-cycle loading condition [15].

\section{A. Failure Mechanisms}

The intrinsic failure mechanisms in power electronics include hot carrier injection and dielectric breakdown and electro-migration, while extrinsic faults consist of latch up, wire lift off, die solder delamination, and substrate solder degradation [16]. These are summarized in Table I [17]. Power electronics modules are subject to two potential failure mode stresses: the first is associated with high electric fields and the second is due to high temperatures. One of the most common defects which is relevant to electric fields is dielectric breakdown [18], [19].

\section{B. Power Cycling Experiment Setup}

IGBTs are designed to work below the nominal characteristic temperature (i.e., $150{ }^{\circ} \mathrm{C}$ ). However, in a railway traction and automotive application, they could experience increased thermal cycling (rise and fall of temperature) which leads to thermo-mechanical stress on the weakened part of the IGBTs packaging interconnections [20]. To simulate this environment in a laboratory set-up, accelerated ageing techniques, such as power cycling, are used. This type of reliability testing can be used to characterize failure mechanisms more accurately than traditional thermal cycling inside an environmental chamber due to the flow of current through the IGBT, and heat dissipation which is similar to what is experienced in application. The induced failure mechanisms depend on the testing conditions and have various levels of degradation similar to real industry applications. Power cycling tests are used to define failure modes and estimate the reliability of standard power electronics module IGBTs [21].

In power cycling tests, the IGBT actively heats up due to the amount of power dissipated at the junction during a constant load cycle. The load cycle also depends on the pull time and change in junction temperature. This results in strain and stress between the aluminium wire and Silicon die, as well as between the die and insulation substrate, where the differences in CTEs (coefficient thermal expansion) initiates cracks at the bonding surface, and creep in the solder joint and substrate layer.

Finally, peel progression of the copper metallization and crack propagation in solder joint lead to the aluminium nitride substrate (AIN) failure mode and wire bond lift off respectively. Highly-accelerated ageing tests use rapid thermal cycling and electrical overstress to speed up prevalent failure mechanisms of the IGBTs in an experiment environment [22]. During ageing tests, electrical parameters and junction temperatures can be simultaneously monitored as precursor parameters for the use of developing IGBT health monitoring capability [23], [24].

The Power Electronic Research Centre in Nottingham University carried out power cycling tests on IGBTs. The test rig used a switched current supplied by a constant current power supply to heat and cool a set of sample "coupons." Each coupon consists of an IGBT die and Bond wires which were attached to a substrate tile (see Fig. 2).

An infrared (IR) sensor is used to monitor the temperature of each die; when the temperature reaches below or above some specified threshold, then the heating current will be 
TABLE I

IDENTIFYING IGBT FAILURE MECHANISMS

\begin{tabular}{|c|c|c|}
\hline Failure Mechanisms & Failure Type & $\begin{array}{l}\text { Failure } \\
\text { Modes }\end{array}$ \\
\hline $\begin{array}{l}\text { Full cycle temperature swings due to power dissipations and self-heating of the wire } \\
\text { bonds }\end{array}$ & Bond wire fatigue & extrinsic \\
\hline Coefficient of Thermal Expansion (CTE) mismatches between wires and silicon & Bond wire lift off & extrinsic \\
\hline Thermo-mechanical stress due to temperature cycles & $\begin{array}{l}\text { Bond wire heel cracking } \\
\text { and fractures }\end{array}$ & extrinsic \\
\hline $\begin{array}{l}\text { Large thermo-mechanical stress and mismatches due to stiffness of the silicon and } \\
\text { materials that cause junction temperature at the centere of the die to rise }\end{array}$ & $\begin{array}{l}\text { Aluminum reconstruction } \\
\text { and metallurgic damage }\end{array}$ & extrinsic \\
\hline $\begin{array}{l}\text { Thermal and thermo-mechanical stress during operation propagates micro-cracks and } \\
\text { aluminum metallization }\end{array}$ & Current leakage & intrinsic \\
\hline Sharp stress level at the pre-existing damage points for brittle materials & Fatigue crack propagations & extrinsic \\
\hline Thermo-mechanical cycling and residual deformation in the bond wires & Corrosion of the wires & extrinsic \\
\hline $\begin{array}{l}\text { High temperature swings and mismatch in the various intermetallic layers with solder } \\
\text { between them }\end{array}$ & Solder fatigue and voids & extrinsic \\
\hline $\begin{array}{l}\text { During transients: High electrical energy produces excessive charging energy in IGBT } \\
\text { which leads to excessive current flow through the gate oxide and high voltage drop } \\
\text { across the IGBT. Results in loss of gate control. }\end{array}$ & $\begin{array}{l}\text { Static and dynamic latch } \\
\text { up }\end{array}$ & intrinsic \\
\hline $\begin{array}{l}\text { Energetic particles in the form of heavy particles, such as electrons, interact with cosmic } \\
\text { neutron rays and burst localized which leads to catastrophic failures. (Cosmic rays) }\end{array}$ & Single event effect & intrinsic \\
\hline
\end{tabular}

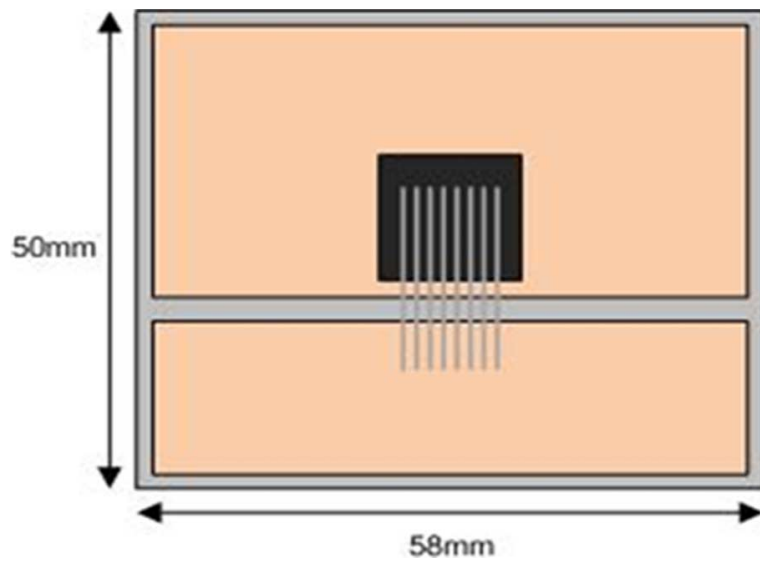

Fig. 2. Test coupon and approximate dimensions.

automatically removed. The temperature is constantly recorded and the collector emitter voltage is measured as a precursor and recorded during each heating cycle. Each of the test beds is controlled independently and they are allowed to heat up until the IR sensor indicates the temperature has reached the upper limit of $120^{\circ} \mathrm{C}$. The bypass switch diverts the heating current

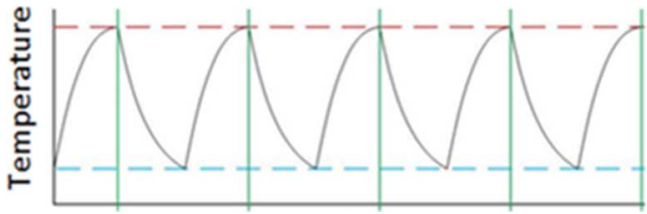

$120^{\circ} \mathrm{C}$

$60^{\circ} \mathrm{C}$

away from the test bed so it starts to cool until the IR sensor indicates that the temperature has fallen to the lower limit of $60{ }^{\circ} \mathrm{C}$, and the bypass switch stops and this power cycling is repeated. The typical temperature waveform during cycling is shown in Fig. 3.

The IGBT accelerated ageing experiment (power cycling) platform is shown in Fig. 4. It was noticed during tests that wire lift off occurred because of thermal expansion mismatches between the wire bond and solder contact points after short-circuit failure.

\section{Ageing Data}

During the prevalent power cycling accelerated ageing process, emitter wire bonds were lifted off and damaged, the 


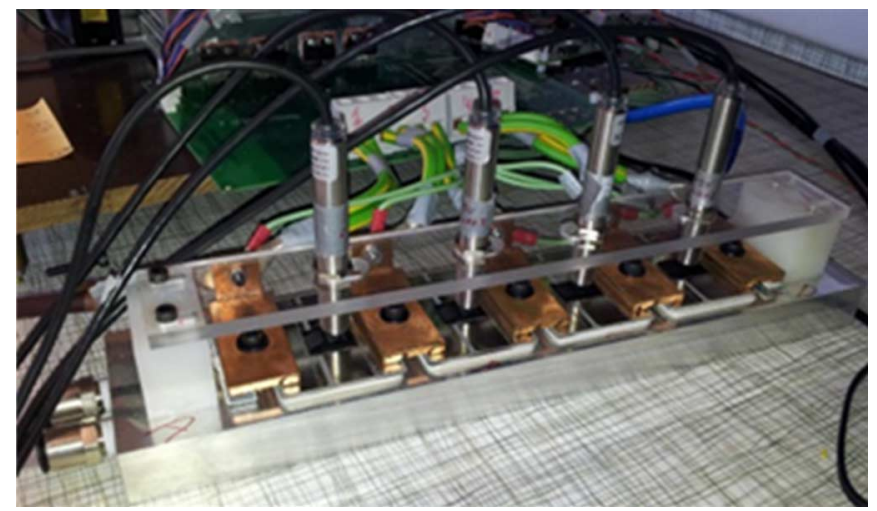

Fig. 4. IGBT power cycling test rig.

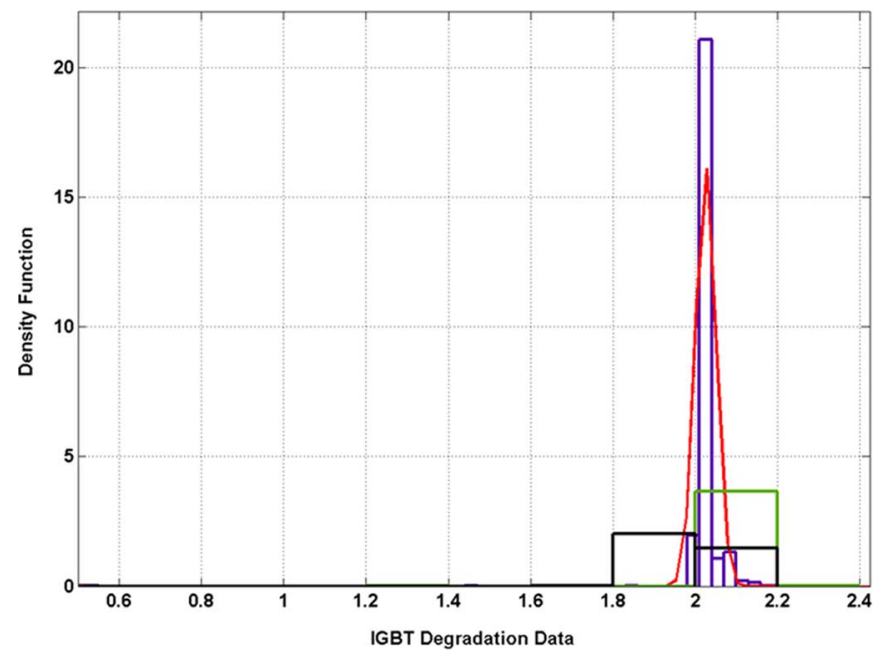

Fig. 5. Normal distribution best fit to IGBT samples.

TABLE II

CHI-SQUARED HYPOTHESIS TESTING

\begin{tabular}{|l|l|l|l|l|}
\hline Probability Distribution & Weibull & Gamma & Poisson & Normal \\
\hline $\mathrm{H}$ & 1 & 1 & 1 & 0 \\
\hline $\mathrm{P}$ & 0.0336 & $1.5169 \mathrm{e}-25$ & $2.4599 \mathrm{c}-13$ & 0.1542 \\
\hline
\end{tabular}

collector emitter voltage on state tracks the degradation and presented as the precursor parameter for developing IGBT prognostics in this paper. During the duration of the degradation process, the $V_{c e}$ (on) measurement shows an increase in non-monotone fashion in discrete steps with noise until the failure of the IGBT. The $V_{c e}$ indicate failure as a quick rise at the end of the ageing process. Data collection successfully has carried out for 22 IGBT samples which can be used promising data mining tool. The raw sensory of time series data trend indicate useful and meaningful pattern to be used for the statistic judgment. The histogram of all samples indicates the Normal distribution is the most appropriate statistical distribution which is shown in Fig. 5; the chi-squared has been used to validate the goodness of fit test in this light, and results are recorded in Table II [25].

It is noted, the null hypothesis $(\mathrm{H}=$ goodness - of-fit to probability distribution function) will

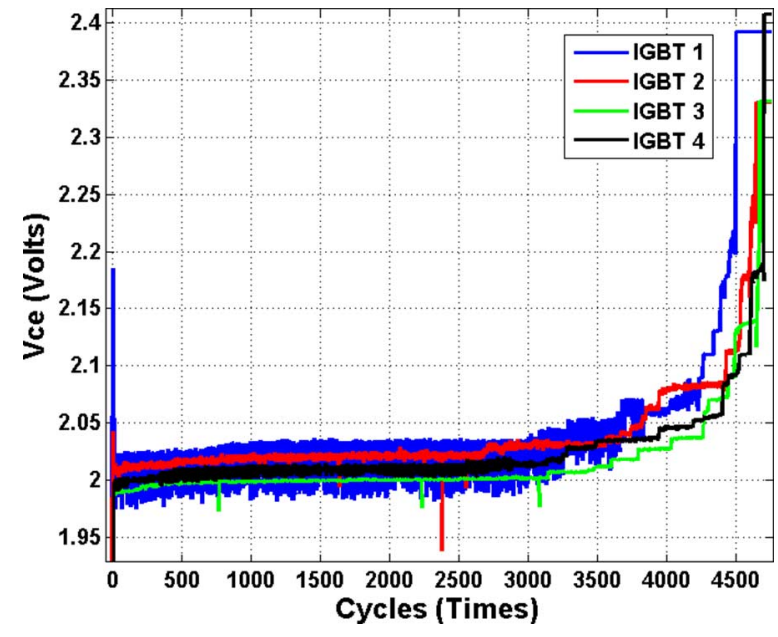

Fig. 6. Four IGBT run-to-failure data set samples.

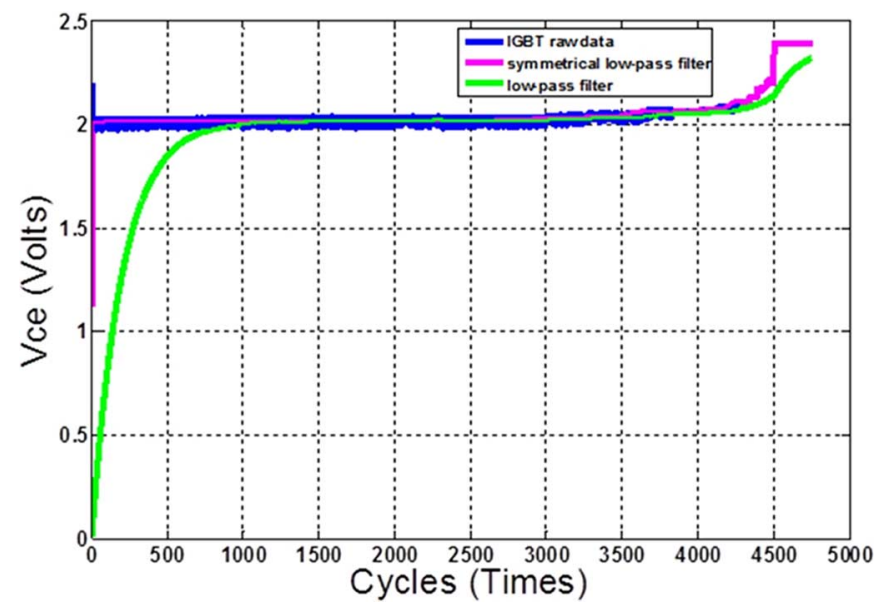

Fig. 7. First IGBT data set after filtering.

be accepted if the $\mathrm{H}$ value is zero and $\mathrm{P}=\mathrm{U}[0,1]$ is greater than the default value of significance level $\alpha=0.05$. To this end, amongst all data sets, the four IGBT raw data sets for simplicity of computation and testing have been chosen, and are shown in Fig. 6; the $y$-axis represents the $V_{c e}$ (voltage) value, while the $x$-axis represents the number of cycles (time), and cycle duration lasts for $6 \mathrm{~s}$.

The first IGBT sample has shown obvious peak, this is due to initial value setting of the PID controller which is input value of the PWM generator. This spike is been eliminated after the first control loop iteration where PID sets its output according to the reference input. The $V_{c e}$ (on) is obtained from a standard power cycling experiment to failure and this will be formatted into a Dimension to be used in data-mining analysis. Initially, the $V_{c e}$ (on) which represents the degradation profile can be filtered using a 1-pole low-pass filter in MATLAB with initial correspond setup according to the data set properties where the time between samples $(\mathrm{T}=1 \mathrm{~s})$ and the filter time constant $(\tau=$ 200 s). As shown in Fig. 7, data filtered by a low-pass filter suffers from delay at the cycles 0 to 1000 due to initial $R C$ delay of 1000 time units. As a solution, a symmetrical low-pass filter (i.e., moving averages filter) is applied to the data set that gives 


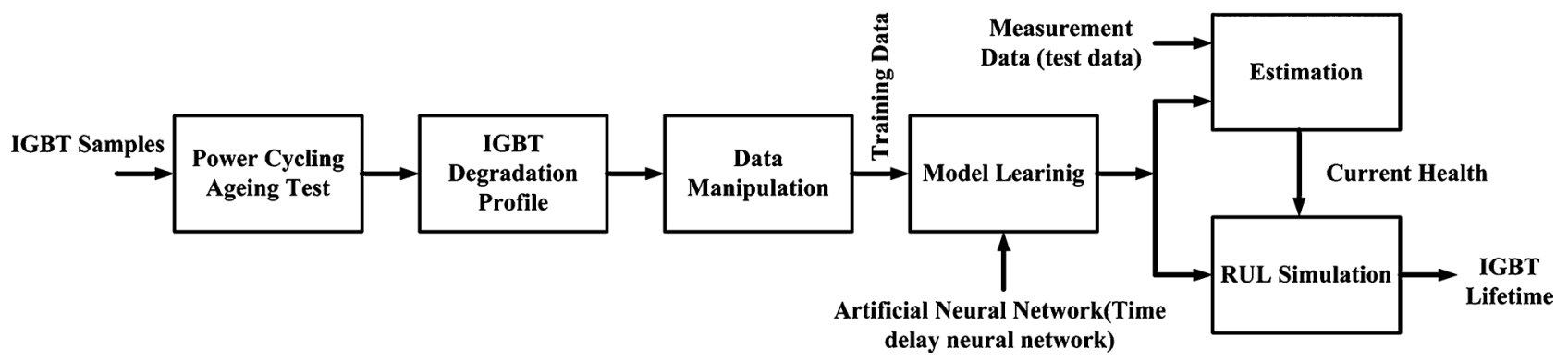

Fig. 8. Outline of the proposed data-mining prognostic approach.

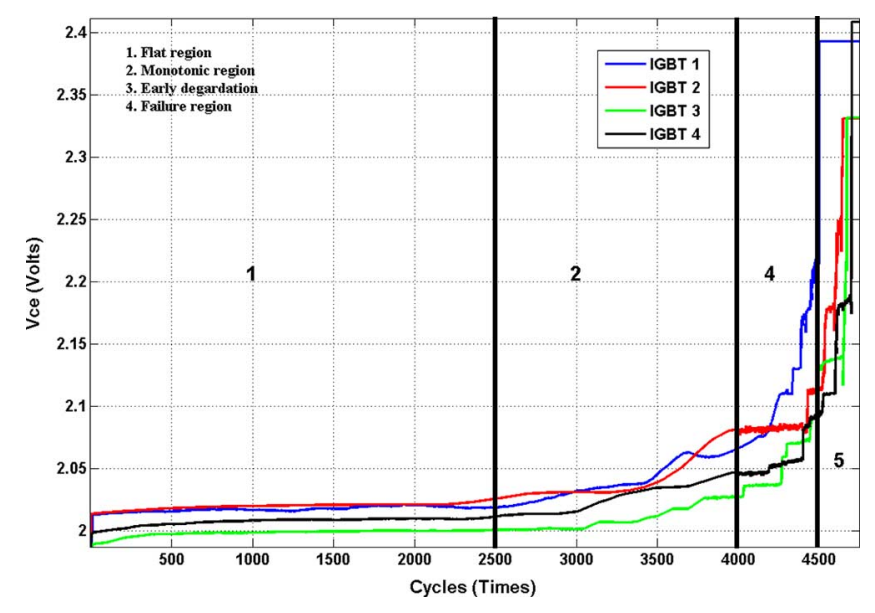

Fig. 9. All IGBT run-to-failure data sets after filtering.

the optimum observation to be employed in a data-mining algorithm [26].

\section{Developing the Degradation Model}

Fig. 8 depicts the overall process of the data-mining algorithm development for the IGBT data sets collected from power cycling experiments. Initially, a model is structured from the training data set using artificial neural networks (e.g., TDNN or neural fuzzy network). Subsequently, the trained model is used with the test data to estimate the current health of the component, this process which continuously tracks the degradation health state of the component and uses as a performance degradation assessment [27]. This is then used with a trapezoidal rule for calculating the area under the estimated curve to predict the remaining useful life of the IGBT (discussed in more detail in the next section). The outline of the algorithm is described in Fig. 8.

\section{A. Discretization of Ageing Data}

Fig. 9 shows the filtered $V_{c e}$ for four different IGBTs utilized in this paper. From this figure, it is noted that the data set is comparatively clear and separated into several discrete states possess different deterioration of the IGBT health states which the following initial life durations are recognized.

1) A flat region where a more and less the ageing process began at a signal amplitude of approximately $2 \mathrm{~V}$ representing the IGBT's healthy condition for duration of 2500 cycles.
2) A monotonic region where the region was characterized by a monotonic increasing trend until reaches discrete manner.

3) An early degradation sign where the degradation process has clearly progressed in increasing discrete steps until IGBT failure has occurred at about $2.4 \mathrm{~V}$.

4) Failed where the IGBT failure life cycle ( 4500 cycles). The life of the IGBT decreases when the abrupt increase in amplitude of the $V c e$ beyond the initially defined amplitude level corresponding to the healthy normal condition. The degradation process is discretized using a uniform quantization process [28] and each degradation state increases about $0.054 \mathrm{~V}$ in a discrete manner which corresponds to one bond wire lift off and is due to one bond wire cut off [47].

The degradation process is characterized by increasing discrete steps of the $V_{c e}$ (from noise-free data) where the step of each degradation phase is uncorrelated to the subsequent phase. Using a quantized cluster validity index, the phase durations of the run-to-failure of four IGBT samples are obtained and recorded as given in Table III. The best number of the failure progression occurs at the tenth health state. Thus, degradation undergoes 10 degradation phases using uniform quantization process for all four IGBT samples which each phase lasts for a period of time before the degradation progresses further to the next phase. And, the genetic algorithm base clustering used to fix 10 cluster centres $\left(C_{1}, C_{2}, C_{3}, \ldots, C_{i}\right)$ which has used the sum of the Euclidean distance of the each cluster centres. For optimization of the natural evolutionary process $D_{i n}=$ $\left[d_{i 1}^{n}, d_{i 2}^{n}, d_{i 3}^{n}, \ldots, d_{i 10}^{n}\right]=\sum_{i=1}^{n} \sum\|d i n-C i\|$ will be minimized of to address the appropriate cluster centre to validate validity index as 10 cluster data sets as shown in Fig. 10 [29].

\section{B. Problem Formulation}

A TDNN model is used in this paper because the IGBT degradation curve of $v_{c e}$ has followed a nonhomogenous sequential dynamic process data which renders the occurrence of a random event, and this can be efficiently modelled into each individual defective voltage. TDNN is well defined for predication of model with having insight dynamic functions. The dynamic of the model is stored using time delayed tap and the nodes updated by using recursive feedback from output to the input at the nodal levels for each iteration. As an advantage, the nonlinear relationship feature of the prediction model that incorporates the input parameters and the output parameters can be eased off efficiently. The TDNN is then adapted to associate the 


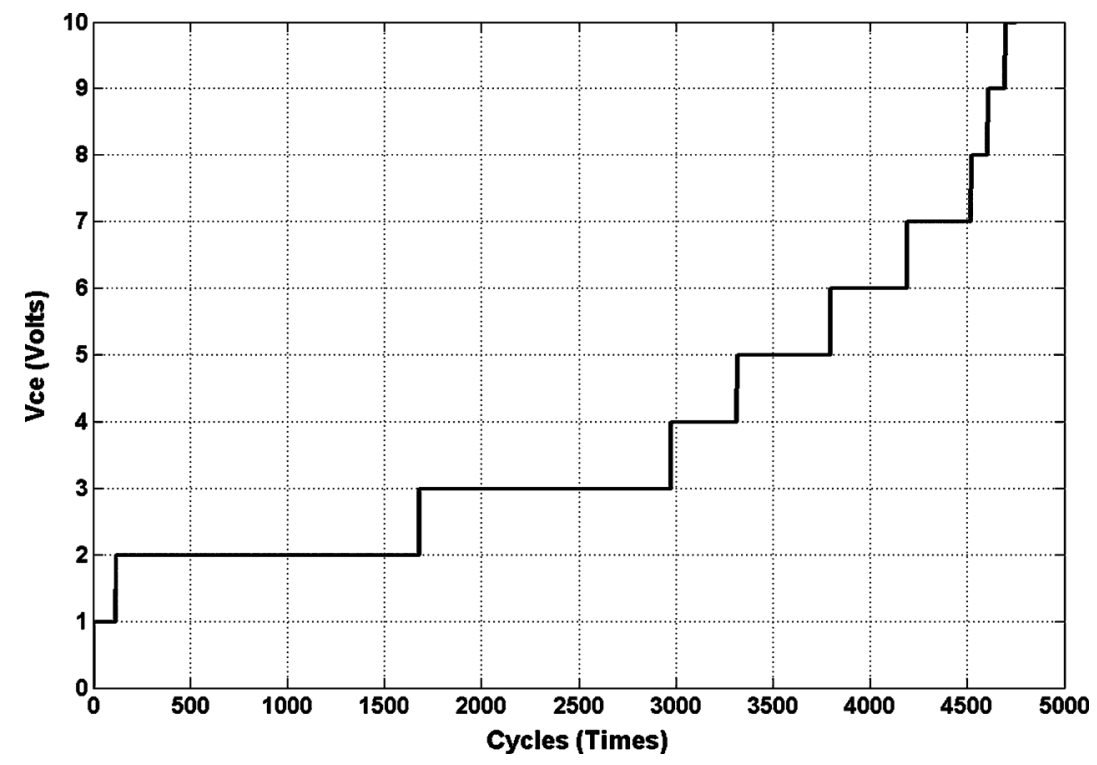

Fig. 10. Degradation phase after optimization process.

TABLE III

IGBT Degradation Phase Duration

\begin{tabular}{|c|c|c|c|c|c|}
\hline \multirow{12}{*}{ 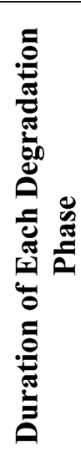 } & IGBT No & 1 & 2 & 3 & 4 \\
\hline & $1^{\text {st }}$ Phase & 1 & 2 & 2 & 2 \\
\hline & $2^{\text {nd }}$ Phase & 109 & 132 & 61 & 117 \\
\hline & $3^{\text {rd }}$ Phase & 1245 & 955 & 1069 & 1561 \\
\hline & $4^{\text {th }}$ Phase & 1440 & 1429 & 1940 & 1296 \\
\hline & $5^{\text {th }}$ Phase & 656 & 866 & 317 & 341 \\
\hline & $6^{\text {th }}$ Phase & 88 & 164 & 645 & 480 \\
\hline & $7^{\text {th }}$ Phase & 521 & 879 & 266 & 394 \\
\hline & $8^{\text {th }}$ Phase & 282 & 94 & 145 & 331 \\
\hline & $9^{\text {th }}$ Phase & 45 & 78 & 209 & 85 \\
\hline & $10^{\text {th }}$ Phase & 111 & 6 & 17 & 91 \\
\hline & IGBT Life & 4498 & 4605 & 4671 & 4698 \\
\hline
\end{tabular}

prediction model with the complex relationship between multivariate inputs and outputs. This is one of the main advantages of the neural network in general is that the physical phenomena of the complex system with nonlinearity dynamics behavior can be neglected and effectively recognize model between multi-dimensional inputs and outputs with unsupervised training. Although, this can be drawback for this technique as the model is unable to interpret the backbone of physical performance of the system [30].

To include dynamic features of degradation processes and failure mechanisms into the model, the drift voltage $\left(\Delta v_{c e}\right)$ of the degradation is considered to be an index of health states, which if not equal to zero, can be presented as wire bond lift off process. The duration of each degradation process is used as an associated failure time $\lambda_{i}^{N_{i}}$ for the input of the TDNN model [31]. The topology of the proposed TDNN model (see Fig. 11) is comprised from one layer for each input and output and one hidden layer with four number of delayed of inputs signals $\left(v_{c e}\right.$, and $\Delta v_{c e}$ ) are introduced to the input layers where the layers are connected with appropriate weights [32].

The proposed TDNN model directly uses $V_{c e}$ (on state) measurement values and the rate of the measurement changed, i.e., dynamic of the actual measurement at current inspection $t$ and previous inspection points $t_{d}=[(t-0),(t-1),(t-2),(t-3)]$ assigned as the inputs time delay. The model takes into account all of these measurement points and changes of the measurement points, all at the same time and at three previous measurement points in order to develop a precise model for prediction of the future health state of the IGBT component in real time. Increasing the number of input nodes would add on more weights and be time-consuming for optimization of the weights. Consequently, model training which has more inspection points would not improve the model's estimation capability.

The neurons' functions are set as $f\left(\sum_{m}\left(a_{m} w_{m}\right)\right)$, where $f$ is the neuron's activation function as sigmoid $\left(1-(1) /\left(e^{x}\right)\right)$ and $w_{m}$ is the synapses' weight associated with the $m$ th input of the neuron in general. Hence, the neuron's output at the hidden layer is constructed in [33]

$$
\begin{aligned}
f_{k p}=\sum_{P=1}^{5} f_{1 p} & \\
=\sum_{p=1}^{5}\left(b_{k p}+\right. & f\left(\left(\sum_{j=0}^{3} w_{(j+1) P} v_{j\left(t-t_{j}\right)}\right)\right. \\
& \left.\left.+\left(\sum_{j=0}^{3} w_{(j+5) P} \Delta v_{j\left(t-t_{j}\right)}\right)\right)\right)
\end{aligned}
$$

as $f_{k p}$ where $\mathrm{K}=\{1,2\}$ is the layer's number $(\mathrm{k}=1$ for hidden, and 2 for output layer); $\mathrm{p}=\{1,2, \ldots, 5\}$ is the neuron number at the hidden layer. The synapses' weight between $j$ th input neuron $(\mathrm{j}=\{1,2, \ldots 8\})$ and the $p$ th neuron at the hidden layer is presented as $W_{j p}$. Considering two sets of delayed inputs from $v$ and $\Delta v$, the neuron's output at the hidden layer is given by (1), where $\mathrm{k}=1$, and time delay $t_{d}$ with $\mathrm{d}=\{0,1,2,3\}$ [34], [35], and $W_{j p}$ is a two dimension matrix constructed from all synapses' weights between inputs and the hidden layer's neurons

$$
W_{j p}=\left[\begin{array}{ccc}
w_{11} & \cdots & w_{15} \\
\vdots & \ddots & \vdots \\
w_{81} & \cdots & w_{85}
\end{array}\right]
$$


$v(t)$

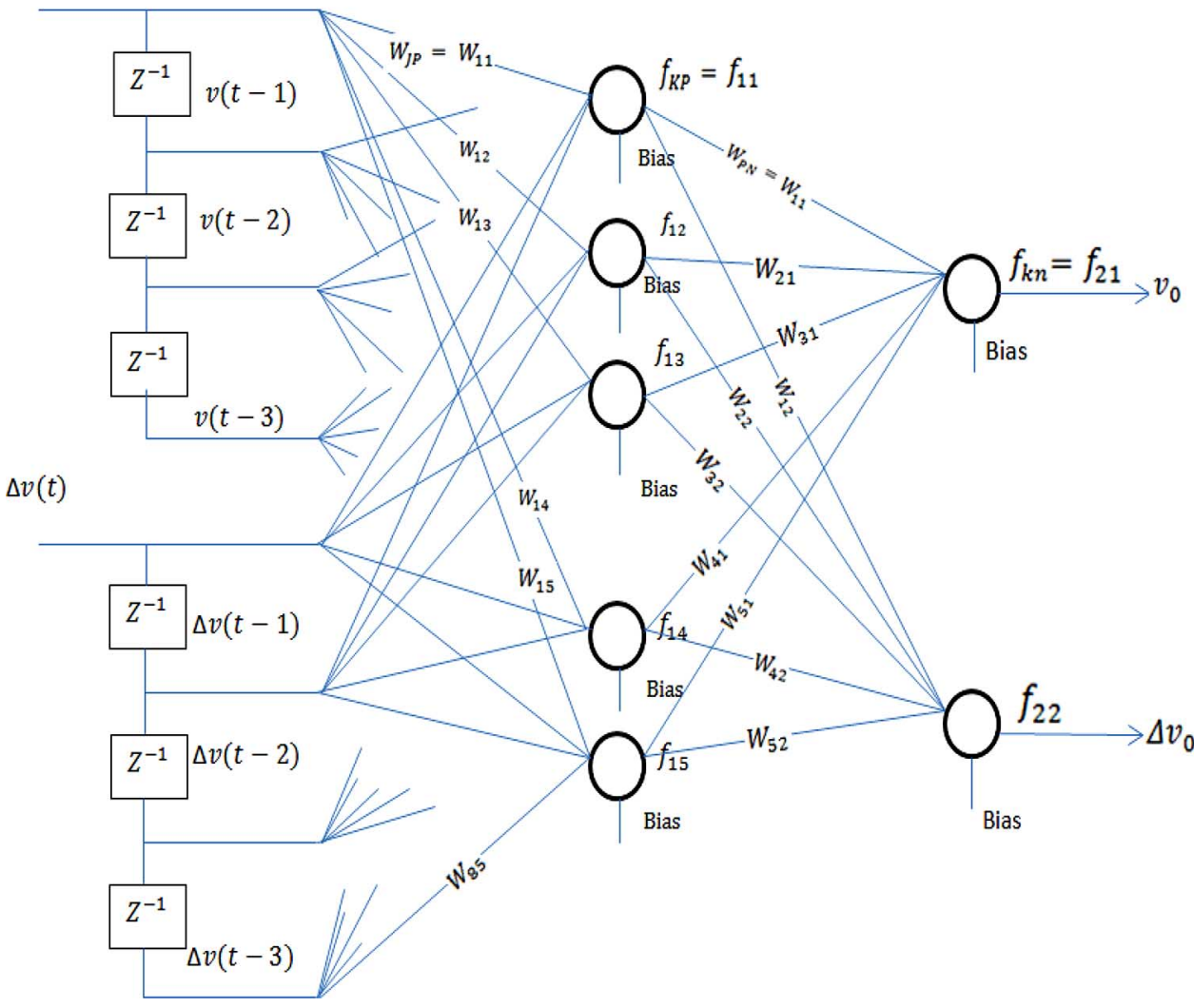

Fig. 11. Structure of the proposed TDNN model.

Similarly, the outputs of neurons, $f_{k n}$, are obtained from (2) where $\mathrm{k}=2, \mathrm{n}=\{1,2\}$ is neuron's number at the output layer, and $W_{P n}$ is the synapses' weight between the pth neuron at the hidden layer and the nth neuron at the output layer. The $f_{k n}$ forms the output model of the TDNN which describes the estimation and dynamic of the process using the initial constructed model [36].

$$
f_{k n}=f_{2 n}=\sum_{p=1}^{5}\left(b_{2 p}+f\left(w_{p n} f_{1 p}\right)\right)
$$

where $W_{p n}$ is a two dimension matrix constructed from all synapses' weights between the hidden layer's neurons and the neurons of the output layer:

$$
W_{p n}=\left[\begin{array}{cc}
w_{11} & w_{12} \\
& \vdots \\
w_{51} & w_{52}
\end{array}\right]
$$

A TDNN topology including all of the synapses weights and parameters of the neurons' activation functions are predefined to fit in the condition monitoring failure measurement. Then the four IGBTs' ageing data set samples are used for training the model using cross validation techniques. During the training process through a number of iterations, the model is adjusted with the weights and biases to converge the output to the desired model and optimization is performed to minimise the error as much as possible to render the best fit prognostic model. To that end, the mean square error (MSE) is used to evaluate the performance of the desired model to computed output values. The model is trained by 150 iteration times, and the MSE incredibly becomes increasingly lower where the final estimation error reaches 0.0333 and the gradients of the output model with respect to weights obtained by $\left(\partial f_{k n}\right) /\left(\partial W_{p n}\right)$ is optimised with Levenberg-Marquardt. The details of the LM for optimization of the TDNN can be found in [37].

\section{Procedure of the Proposed TDNN Method}

The procedure of the proposed TDNN method is shown in Fig. 12. The explanation of the flowchart of the proposed algorithm is provided below [38], [39]. The flowchart is executed with the three following steps: 1) data manipulation; 2) model training; and 3) propagation.

Step 1: We start from the available run-to-failure IGBTs' historical data, which includes the degradation process values from the precursor parameter $V_{c e}$ at inspection points for the power cycling ageing measurement. The low-pass filter is used to filter the noises that are populated inside the data set. The duration of the degradation phase for all data sets is optimized using maximum likelihood estimation, the results of which will be used for the classification using K-mean clustering on the data set. The threshold value is generally set based on expert knowledge in this case at three times greater than the standard deviation of the flat region where the degradation exponentially starts to rise with a rapid gradient. This almost $\pm 15 \%$ deviates from its original value [46]. In this data set, we attempted to set the threshold at 7 which is almost the critical discrete level which represents 


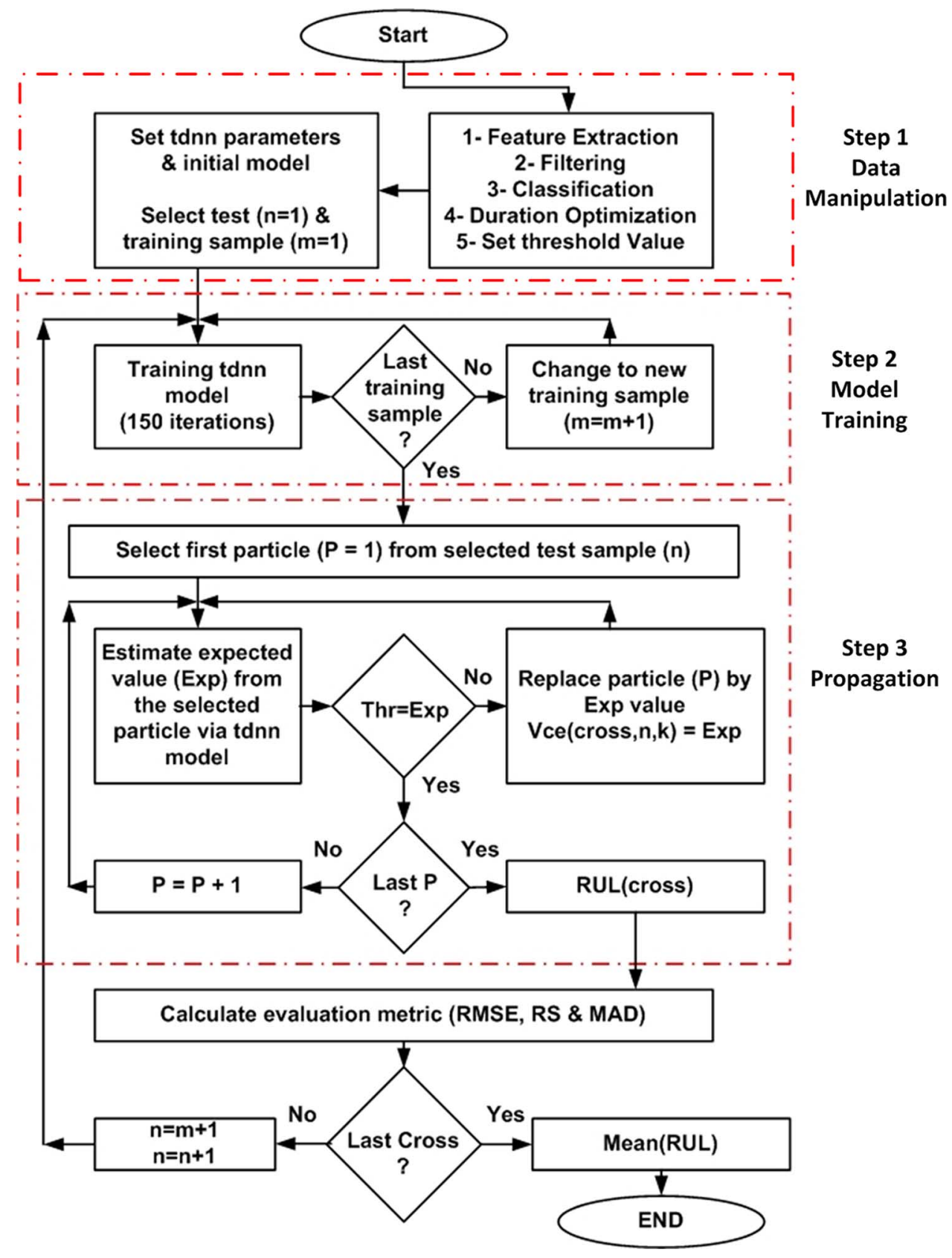

Fig. 12. Algorithm of the proposed TDNN method.

the catastrophic degradation profile in order to validate the prognostic model and algorithm. The classified data for a failure history is used for the development of a TDNN to estimate the future health state of the IGBTs in real time. The model starts to build up with two input parameters $v_{c e}$ and $\Delta v_{c e}$ in conjunction with two hidden layers, where the first hidden layer includes fiveneurons and the second layer contains two neurons. The two input parameters are assisted with three tapped delay lines as embedding local memory into both the input and hidden layers which provides the dynamic ability to the model structure. The first data set $(\mathrm{m}=1)$ is peaked up for training the TDNN model and the second data set $(\mathbf{n}=1)$ is chosen as a test data for model validation [40].
Step 2: Training the TDNN model is a stochatic process and depends on the initial weights and is adjusted using the LM method which also requires the training data set and the testing data set. The training of the model is conducted using time series data and the difference in time related to the pattern, to estimate the number of the sequence of states that corresponds to the current observed health state for 150 iterations. The training process is completed using cross-validation techniques surely to overcome dissatisfaction of availability of a lower number of data sets; therefore, all datasets are partitioned for use for both training and testing which limits the overfitting model with the training data set. Before the data set reaches the last data set (Cross Number) for testing and the last training sample is checked, then, if the Cross Number is remaining, the learning 


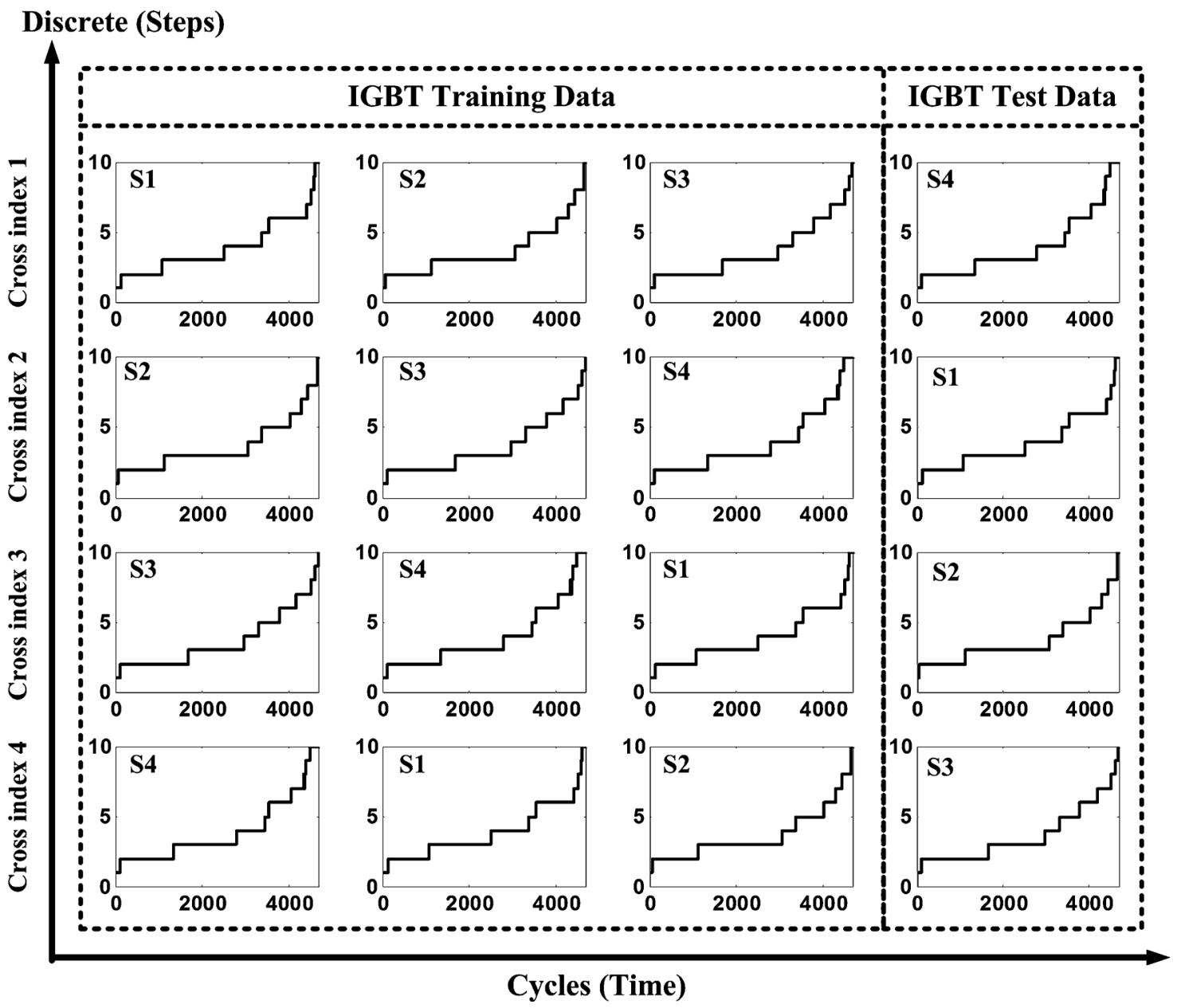

Fig. 13. TDNN training and testing modelining for IGBTs failure data set.

algorithm continues to simply run both the training and the testing sample increment by one. This process repeats until four failure histories to construct the TDNN training model and validation of the model are completed (see Fig. 13 for the cross validation; cross $=1, \ldots, 4)$. Four failure history data sets are divided into three training sets and one testing set; the model learns from three samples and validated with one sample and the learning model will replicate once the first sample becomes the last sample/last cross in the algorithm for validation. The model learns to estimate the failure degradation phase, but, if the current health state based on the measurement point needs to be identified, then the mean of the RUL results from each of validation represents the final results.

Step 3: The end-of-life (EO4L) of the IGBTs can be calculated by peaking up the first particle from the selected data set as the measurement value at the current state which will be propagated through the degradation model until the threshold value. The availability of the new inspection particle will be checked out once the estimation value reaches the predefined threshold value if the new inspection particle is available. Therefore, the number of particles will be incremented by one until the last particle. The propagation process continues when the measurement point is greater than the length of the last particle and it then moves to calculate the RUL and then the prognostic evaluation metric, such as RMSE, RS, and MAD. If the expected value or estimated value has not reached the threshold value, then the current inspection particle will be updated by an estimated value in the measurement parameter as the recursive algorithm progressing the degradation phase until it reaches a significant promising threshold state. The LM algorithm for the TDNN training is run four times, and the trained TDNN corresponding to the lowest prediction performance mean square error (MSE) after 626 epochs is $3.9805 \mathrm{e}-06$. The prediction performance is shown in Fig. 14, which almost is mapping the test data (e.g., input measurement data) to the train TDNN model.

The degradation duration for the estimation model is quite random for each testing model and the number of degradation phases varies slightly. The estimation degradation is assumed to have followed a nonhomogenous probability distribution process which renders the occurrence of random events. There are two major types of probability distributions: distribution with mean $(\lambda)$ and standard deviation $(\delta)$, i.e., normal, Gaussian, exponential, Weibull distributions; and distributions with solely $\delta=\lambda$, i.e., Poisson, binomial distributions. As this paper is limited to only four data set; it cannot be confirmed which distribution suits to the IGBT failure data set. In [26], two candidates distribution (e.g., Gamma and Poisson) have been used against data set from each types. Given the fact that all samples are comparatevily continuous time series dataset, therefore as a solution for instance, normal from first type 
TABLE IV

MLE PARAMETERS OF ESTIMATED DURATION

\begin{tabular}{|c|c|c|c|c|c|c|c|c|c|c|}
\hline Parameters & Model 1 & Model 2 & Model 3 & Model 4 & Model 5 & Model 6 & Model 7 & Model 8 & Model 9 & Model 10 \\
\hline $\boldsymbol{\mu}$ & 1.75 & 104.75 & 1207.5 & 1526.25 & 545 & 344.25 & 515 & 213 & 104.25 & 56.25 \\
\hline $\boldsymbol{\delta}$ & 0.5 & 30.685 & 264.138 & 283.491 & 263.920 & 262.699 & 264.054 & 111.758 & 71.978 & 52.506 \\
\hline
\end{tabular}

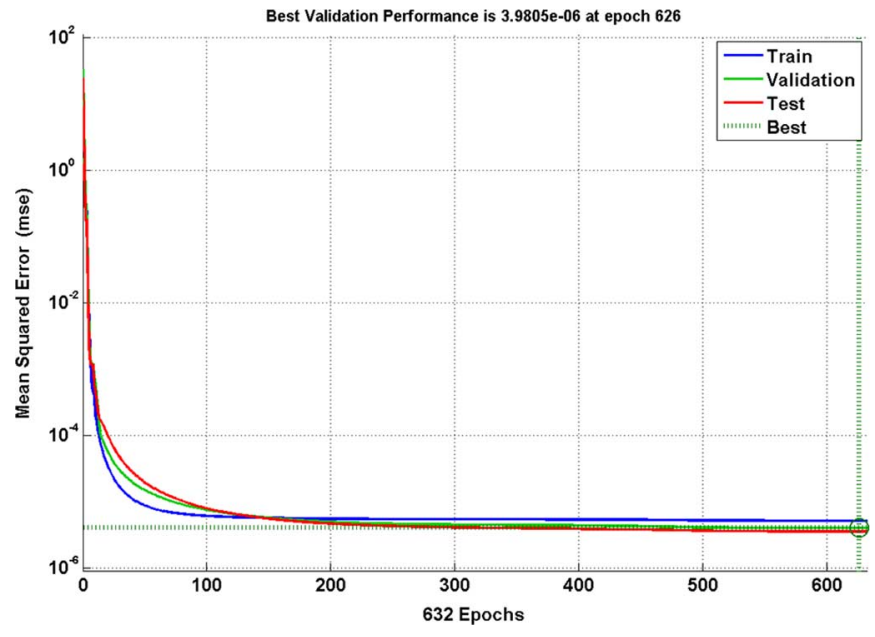

Fig. 14. Performance of the proposed TDNN.

is selected. Results using Gamma and Poisson distributions are presented in [26]. In this paper, we present results from normal distribution and compare it with Gamma and Poisson distributions, and their validity is evaluated in Section VI. In a normal distribution, mean $\mu$ and variance $\sigma^{2}$ parameters [cf. (3)] are estimated in order to obtain the best fit probability distribution function for each degradation phase.

\section{Prognostic ApProACH}

\section{A. Degradation Model Estimation Optimization}

Maximum likelihood estimation (MLE) is used as an objective function to maximize the density probability function (i.e., normal distribution function). A Normal distribution as shown in

$$
y=f\left(x_{i} \mid \mu, \sigma^{2}\right)=\left[\frac{1}{\sqrt{2 \pi \sigma^{2}}} e^{\frac{-(x-\mu)^{2}}{2 \sigma^{2}}}\right], \quad x_{i} \geq 0
$$

is the probability distribution of the IGBT estimated degradation phase $\left(x_{i}\right)$ given normal parameter $\mu$ and $\sigma^{2}$ [41]: The MLE method is used to estimate the underlying rate parameters' sample mean $\mu$ and the variance $\sigma$ for the normal process in order to generate these counts. The first step is to write the joint probability mass function; in this case, $x_{i}$, because it is a discrete random variable of the positive integers [42]. Since all discrete random variables are independent, the product of the individual density functions can be obtained. The next step is to calculate the maximum of this probability mass function with respect to $\mu, \sigma$; however, simplifications in [43] make it possible to find the maximum of the probability mass function from maximum of $\mu, \sigma$ in the form of

$$
\begin{aligned}
\widehat{\mu_{n}} M L E & =\frac{1}{n} \sum_{j=1}^{N} x_{n} \\
{\widehat{\sigma_{n}}}^{2} M L E & =\frac{1}{n} \sum_{j=1}^{n}\left(x_{j}-\hat{\mu}\right)^{2} .
\end{aligned}
$$

The maximum-likelihood criterion is a fairly general one and also a fairly powerful to show the true value (the maxima) in close form algebraically. The true value $\hat{\lambda} M L E$ is the most probable one that generates maximum likelihood data. Using the analytical maximum-likelihood estimation (MLE) method to estimate the best fit of the modelling parameter, the estimator $\hat{\mu}$ equals the sample mean and the estimator $\hat{\sigma}^{2}$ equals the unadjusted sample variance for a normal distribution [43]. According to Table II, the number of health states for each of the four components' health estimation is ten nonhomogenous discrete phase durations. The MLE function is employed for each health state (HS) of the four components as in

$$
\begin{aligned}
& \mu_{(H S)_{j}=\frac{1}{n} \sum_{n=1}^{4} \sum_{j=1}^{10} H S_{j}} \\
& \quad j=1, \ldots, 10 \text { number of health states } \\
& \quad n=1, \ldots, 4 \text { number of IGBTs components } \\
& \sigma_{(H S)_{j}=\frac{1}{n} \sum_{n=1}^{4} \sum_{j=1}^{10} H S_{j}}
\end{aligned}
$$

and the results are presented in Table IV [28].

\section{B. RUL Simulation Algorithm}

RUL estimation and its related simulation algorithm are processed based on the fact that the maximum estimated life time of the IGBT is divided into a number of estimated durations. Failure at each estimated duration is progressed in a different manner which results in different rates of degradation known as estimated degradation phases $(\tau)$, as discussed in the previous section, (see Fig. 13. Estimated degradation phases result from the TDNN model, and presents number of health states that IGBT may experience in real time. Considering $\mathrm{n}$ number of $\tau$, the life of a health state is calculated in normalized form (in respect to summation of $\tau$ ) using the following equation:

$$
H S L_{i}=t_{\mathrm{thr}} \cdot \frac{\tau_{i}}{\sum_{j=1}^{n} \tau_{j}}, \quad i=\{1,2, \ldots, n\}
$$

where $\mathrm{HSL}_{i}$ presents a period that $i$ th health state life will last for a number of cycles between a starting cycle $\left(t_{s}\right)$ and an ending cycle $\left(t_{n}\right)$; and $t_{\mathrm{thr}}$ is the maximum real life time of the IGBT that is not necessarily equal to the total $\tau$, because total $\tau$ 
is accumulation of estimated values. A normal probability distribution function $(f(t))$ is assigned to the each HSL wherein the center and variance of $f(t)$ are the mean of cycles occurring in the $H S L_{i}\left(\left(t_{s}+t_{n}\right) / 2\right)$ and the difference of the maximum and the minimum cycles of the $H S L_{i}\left(t_{s}-t_{n}\right)$, respectively. As each HSL has an area of 1 and different variance values, accordingly, HSLs will have different maximum probabilities. A per-unit HSL is obtained by the following equation:

$$
H S L_{p u_{i}}=\frac{f_{i}(t)}{\sum_{k=1}^{n} \int_{t_{0}}^{\tau} f_{k}(t)}, \quad i=\{1,2, \ldots, n\}
$$

where $\mathrm{HSL}_{p u i}$ is the $i$ th HSL in per-unit form, $f_{i}(t)$ is the normal probability distribution function for the $i$ th estimated degradation duration, and $t_{0}$ is the operation time at measurement point. The probability of estimated duration for each inspection time (PDLT) is

$$
P D L T_{i}=H S L_{p u_{i}} \cdot \tau_{i}, \quad i=\{1,2, \ldots, n\} .
$$

By means of PDLT, RUL calculation for IGBT components is performed to calculate the probability of each measurement particle that occurs in each HSL of the component using a normal probability density function. The area of each HSL sweeps through as the degradation progresses from the beginning of the IGBT up until the end of life

$$
\begin{gathered}
\mathrm{RUL}=\sum_{i=1}^{p} \sum_{j=1}^{k}\left(P D L T_{i, j}\right) \\
i=1, \ldots, p=\text { number of duration lives } \\
j=1, \ldots, k=\text { number of particles } \\
\text { for each duration life }
\end{gathered}
$$

The process of the RUL calculation is given in the flowchart of the proposed algorithm in Fig. 15. The approach depends on the IGBT failure model estimation and the normal distribution based on the optimized duration parameter. A time delay neural network approach for the degradation modeling is constructed and sweeping the estimation value under the normal distribution curve for each estimated degradation phase $\left(\tau_{i}\right)$ until the end of the component's life (EOL) or it can be swept up to a predefined threshold value. Then, the RUL (i.e., mean and confidence levels) is calculated using the distribution of estimated values based on the area of each estimated degradation phase by MC simulation.

Fig. 16 shows an example (i.e., IGBT sample number 4) of $H S L_{p u}$ results. The results were computed based on a normal distribution model which shows the area under each curve equivalent to one unit per duration. The oscillated blue, red and green curves show the mean of the area per unit, $10 \%$ and $90 \%$ deviation confidence bounds of the area per unit, respectively. The oscillations present the degradation process and the RUL predictions are also carried out at each moment of the degradation process. Hence, the RUL prediction occurs throughout the whole process of the IGBT degradation experiment. For instance, the observation peaks at measurement 1500 cycles (cycle per second) where the black area under the each

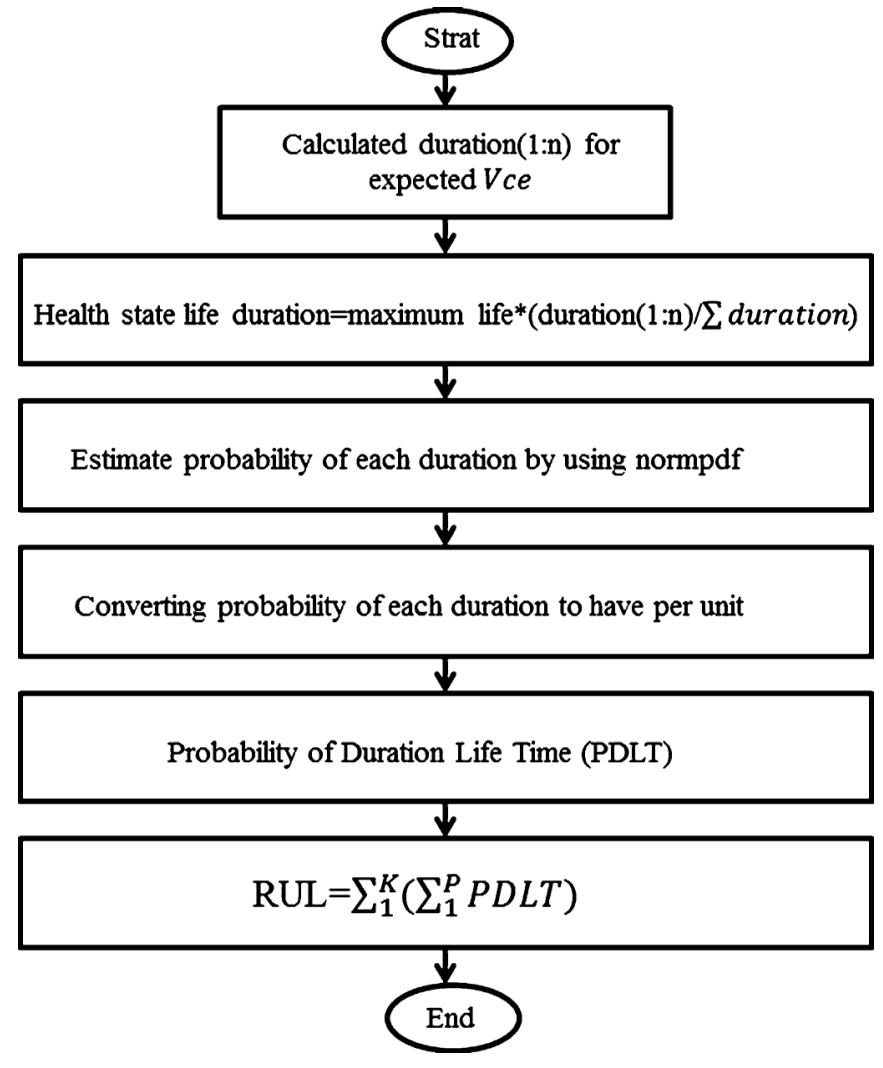

Fig. 15. RUL calculation approach algorithm.

oscillation cycle presents the duration life per unit till it reaches its maximum life which is slightly above 4000 cycles (Fig. 16).

\section{RESULTS}

\section{A. RUL Simulation Results}

The RUL prognostic results which have been estimated by the TDNN and fused with the probabilistic approach to reflect the discrete change in the degradation state are expressed by a series of polylines. The prognostic simulation is a process from the beginning of the healthy state condition of the IGBT running to the threshold value at 7 . The $V_{c e}$ as a degradation indicator is monitored, and the relevant sensor data during the on state are recorded at each cycle where the degradation related to the packaging failure mode has caused the IGBT to fail.

Fig. 17 shows four IGBT samples of RUL simulation results. The RUL result is computed using the statistical approach based on the estimation results from the TDNN failure model. The results are promising for early failure findings and improve decision-making based on confidence levels. The straight black, red and green oscillatory lines are used as the real RUL and its 10 and 90 percentile deviation confidence bounds, respectively. These three lines present the accuracy of the prognostic estimation during each degradation transient. The blue plot indicates the mean value of the RUL simulation. At the beginning of the RUL rendering, all prediction plots diverge from the real RUL value. As the operating time is rendered toward the end of the IGBT life, the 10 and 90 percentile confidence bounds significantly converge to the real RUL value where the accuracy of the life estimation is vital rather than at the beginning of the device 


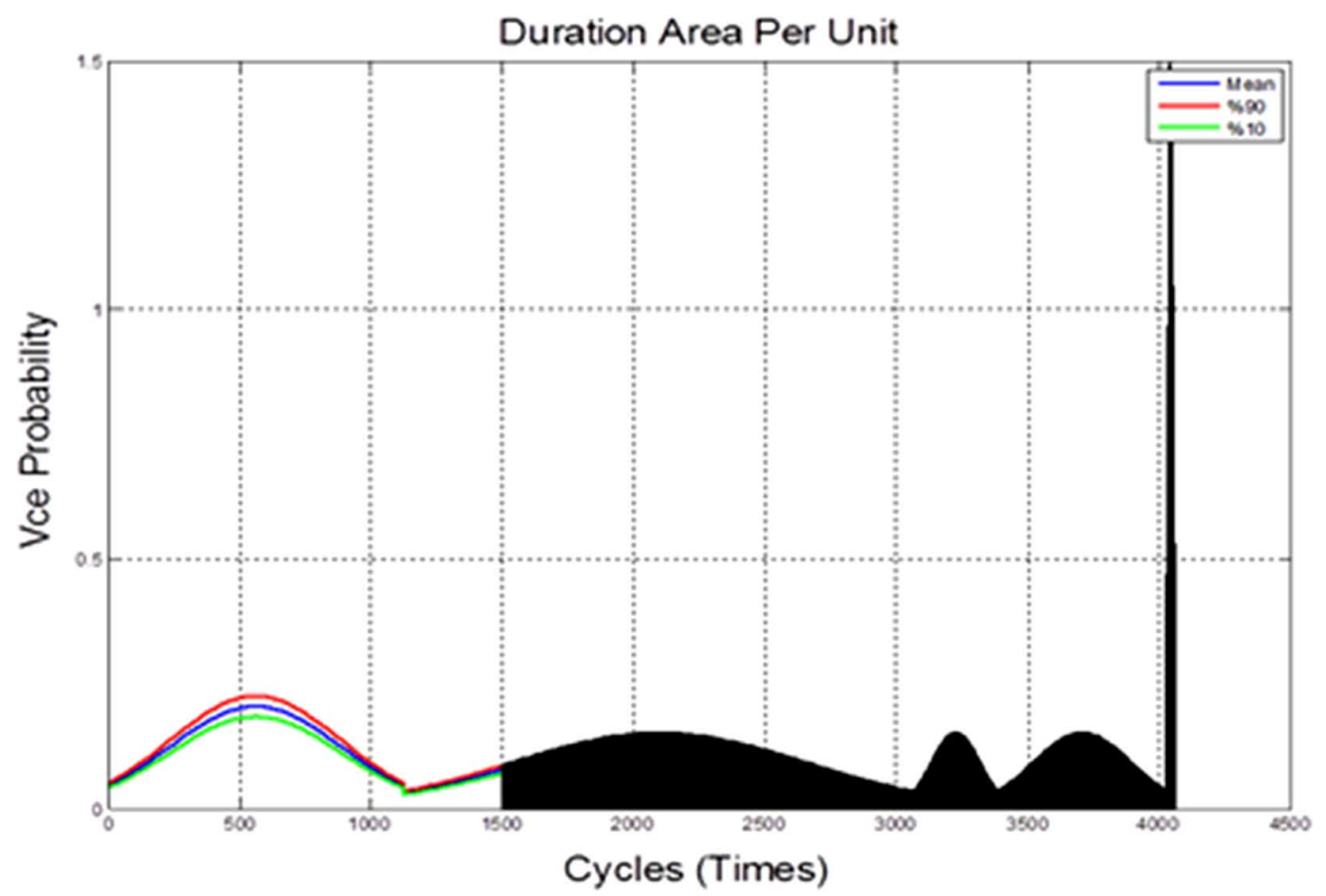

Fig. 16. Normalization of the duration life.

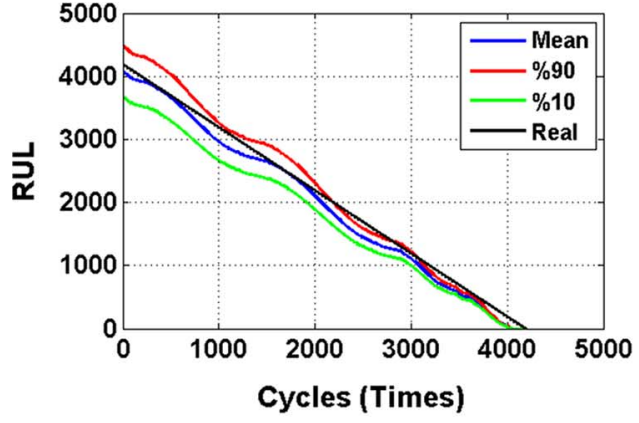

(a)

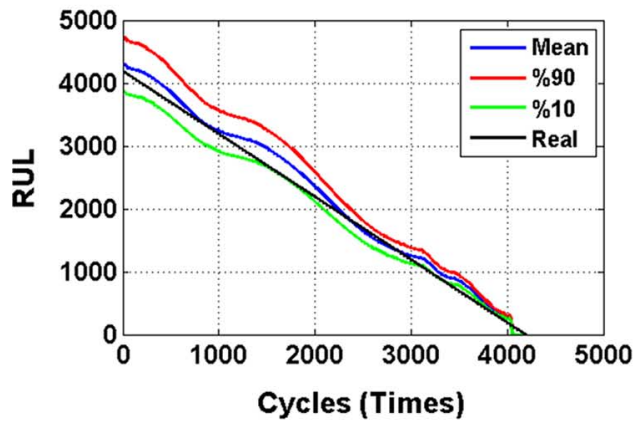

(c)

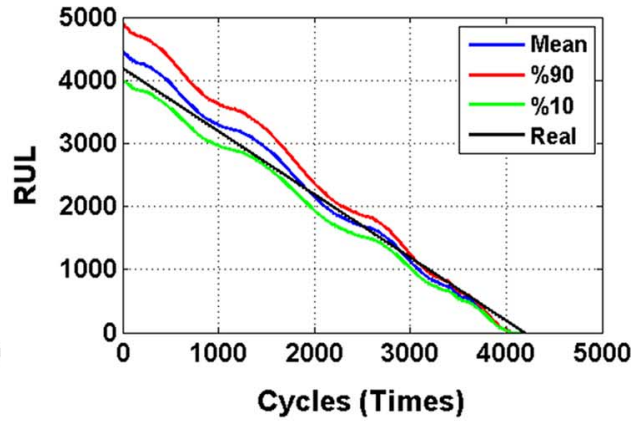

(b)

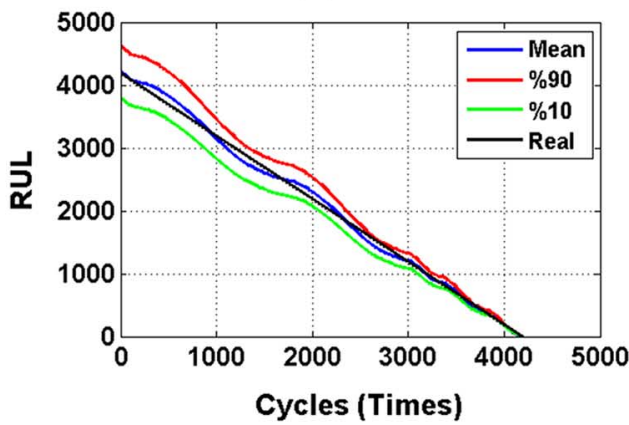

(d)

Fig. 17. RUL of TDNN failure model using normalization techniques.

life. The confidence bounds initially offer meaningful information as the normal distribution associated with mean and standard deviation. However, the confidence bounds lie very close to the mean value as the degradation process reaches the end of the ageing process.

\section{B. RUL Estimation Error}

In this paper, the RMSE and RA are used to assess the precision of the proposed prognostic performance for all duration of failure progression, and the results are compare against an extended KF [11]. Basically, RMSE measures the error between the real and predicted values while prediction is performed by a model or an estimator [44]. Due to the nature of degradation data that may widely differ from one sample point to the next 
TABLE V

RMSE Prognostic Performance Metric for All igBt Samples Degradation Phase

\begin{tabular}{|c|c|c|c|c|}
\hline Degradation Phase & IGBT 1 & IGBT 2 & IGBT 3 & IGBT 4 \\
\hline 1 & 1.15 & 1.15 & 1.16 & 4.28 \\
\hline 2 & 1.10 & 1.12 & 0.85 & 4.14 \\
\hline 3 & 0.89 & 0.93 & 0.84 & 3.58 \\
\hline 4 & 0.90 & 0.90 & 0.87 & 3.45 \\
\hline 5 & 0.94 & 0.87 & 0.91 & 3.28 \\
\hline 6 & 0.97 & 0.84 & 0.91 & 3.17 \\
\hline
\end{tabular}

TABLE VI

RA Prognostic Performance Metric of All igBt Samples Degradation Phase

\begin{tabular}{|c|c|c|c|c|}
\hline Degradation Phase & IGBT 1 & IGBT 2 & IGBT 3 & IGBT 4 \\
\hline 1 & 99.9703 & 99.9433 & 99.9738 & 99.9994 \\
\hline 2 & 99.9774 & 99.9466 & 99.9747 & 100.00 \\
\hline 3 & 99.9905 & 99.9380 & 99.9579 & 99.9912 \\
\hline 4 & 99.9924 & 99.9245 & 99.9487 & 99.9747 \\
\hline 5 & 99.9939 & 99.9233 & 99.9492 & 99.9714 \\
\hline 6 & 99.9940 & 99.9249 & 99.9511 & 99.9678 \\
\hline
\end{tabular}

sample, mainly where wire bonds lifted off, RA might provide us with a more accurate metric to evaluate the performance of prognostic model [11], [45].

If the $y_{i}^{\wedge}$ is the estimated RUL, and $y_{i}$ is the real RUL value, then the RMSE and RA can be calculated using

$$
\begin{aligned}
\mathrm{RMSE} & =\sqrt{\frac{1}{n} \sum_{i=1}^{n}\left(y_{i}^{\wedge}-y_{i}\right)^{2}} \\
\mathrm{RA} & =100\left(1-\frac{\left|y_{i}-y_{i}^{\wedge}\right|}{y_{i}}\right)
\end{aligned}
$$

respectively. The performance of the proposed prognostic technique is summarized in Tables V and VI using RMSE and RA metrics for each life duration, respectively. Table VII also compares the RMSE values of the proposed technique with the RMSE values from [26] that employ Gamma and Poisson probability distributions in a Markov chain probabilistic approach. It is observed from metric that the proposed fusion technique has considerably improved prediction for all degradation phase process compare to just stochastic model base approach. In general, hybrid approach is proved to be a versatile approach for prediction process and RUL calculation also, presents better RMSE values. Furthermore, it has significant decision making because the 90th and 10th percentiles confidence width bonds are narrow realistically close to the mean during the whole prediction and do provide meaningful information for decision-makers due the fact that most of the occurrence of the estimated failure data is disseminated within standard deviation According to the RA values based on the proposed approach, the IGBT test sample number 1 has the largest RA value; therefore, it presents a more accurate predictive failure model amongst all four IGBTs, and can be employed as a precise prognostic model. From [11], extended KF-based prognostics presents maximum RA as a significant prognostic performansce is 97.052 , it can be noted from Table VI, RA incredibly gives better results for all four samples, averaging 99.9 based on our approach.
TABLE VII

Prognostic Performance Metric

\begin{tabular}{|c|c|c|c|c|}
\hline RMSE \% & IGBT 1 & IGBT 2 & IGBT 3 & IGBT 4 \\
\hline Normal & $0.9917 \%$ & $0.9683 \%$ & $0.9233 \%$ & $3.65 \%$ \\
\hline Poisson & $28.6 \%$ & $20.0 \%$ & $25.8 \%$ & $27.2 \%$ \\
\hline Gamma & $33.17 \%$ & $33.97 \%$ & $34.85 \%$ & $33.19 \%$ \\
\hline
\end{tabular}

\section{CONCLUSION}

The main contribution of this paper is the development and implementation of a TDNN failure model of IGBT for indication of the current health state and fuses with the principle of the area under the curve for RUL calculation. To this end, the area of the breaking region of the failure model is fitted with a probabilistic distribution function (i.e., normal distribution function). In addition, Monte Carlo simulation is utilized in the algorithm to generate the calculation area up until the threshold value to approximate the IGBT's RUL. Overall, the IGBT degeneration model is built based on IGBT failure mechanism and degradation characterization. The stochastic approach and Monte Carlo simulation are used to calculate the area under the estimated degradation phases and the precursor parameter, collector emitter voltage $\left(v_{c e}\right)$, is integrated to develop the prognostic algorithm for predicting the IGBT's RUL. Comparison with the results of RUL prediction is shown in Tables $\mathrm{V}$ and VI which show all four samples. The first samples present significantly small RMSE and large RA values. The implementation of the developed prognostic framework could be applied to provide advance warning of failures, thereby preventing costly power electronic system downtime and failures. The TDNN failure model can perform much more efficiently when it fuses with the statistical approach for failure RUL prediction results in 
some IGBTs. The combined model in this paper is only based on the normal distribution, established and implemented in IGBT RUL prediction.

\section{REFERENCES}

[1] L. Zhang, X. Li, and J. Yu, "A review of fault prognostics in condition based maintenance," Proc. SPIE, vol. 6357J, pp. 1-6, 2006, Art. ID 635752.

[2] E. Corchado, M. G. Romay, and A. M. Savio, Hybrid Artificial Intelligence Systems. San Sebastian, Spain: Springer, 2010.

[3] M. Samie, S. Perinpanayagam, A. Alghassi, A. M. S. Motlagh, and E. Kapetanios, "Developing prognostic models using duality principles for DC-to-DC converters," IEEE Trans. Power Electron., vol. 30, no. 5, pp. 2872-2884, May 2015.

[4] H. Wang, M. Liserre, F. Blaabjerg, P. de Place Rimmen, J. B. Jacobsen, T. Kvisgaard, and J. Landkildehus, "Transitioning to physics-of-failure as a reliability driver in power electronics," IEEE J. Emerging Sel. Topics Power Electron., vol. 2, no. 1, pp. 97-114, Mar. 2014.

[5] J. R. Celaya, P. Wysocki, V. Vashchenko, S. Saha, and K. Goebel, "Accelerated aging system for prognostics of power semiconductor devices," in Proc. IEEE AUTOTESTCON, 2010, pp. 1-6.

[6] R. M. Crowder, "Electrically powered actuation for civil aircraft," in Proc.. IEE Colloq. Actuator Technol.: Current Practice and New Developments, 1996, pp. 5/1-5/3, Dig. 1996/110.

[7] X. Si, W. Wang, C. Hu, and D. Zhou, "Remaining useful life estimation-A review on the statistical data driven approaches," Eur. J. Oper. Res., vol. 213, no. 1, pp. 1-14, 2011.

[8] K. Medjaher, D. A. Tobon-Mejia, and N. Zerhouni, "Remaining useful life estimation of critical components with application to bearings," IEEE Trans. Rel., vol. 61, no. 2, pp. 292-302, Feb. 2012.

[9] J. Durbin and S. Koopman, Time Series Analysis by State Space Methods, 2nd ed. Oxford, U.K.: Oxford Univ., 2012.

[10] N. Patil, D. Das, and M. Pecht, "A prognostic approach for non-punch through and field stop IGBTs," Microelectron. Rel., vol. 52, no. 3, pp. 482-488, 2012.

[11] J. R. Celaya, A. Saxena, C. S. Kulkarni, S. Saha, and K. Goebel, "Prognostics approach for power MOSFET under thermal-stress aging," in Proc. Annu. Rel. Maintain. Symp., 2012, pp. 1-6.

[12] B. Saha, J. R. Celaya, P. F. Wysocki, and K. F. Goebel, "Towards prognostics for electronics components," in Proc. IEEE Aerosp. Conf., 2009 , pp. 1-7.

[13] M. Musallam, C. M. Johnson, C. Yin, H. Lu, and C. Bailey, "In-service life consumption estimation in power modules," in Proc. 13th Int. Conf. Power Electron. Motion Control, Poznan, Poland, 2008, pp. 76-83.

[14] S. Kumar, M. Torres, Y. C. Chan, and M. Pecht, "A hybrid prognostics methodology for electronic products," in Proc. IEEE Int. Joint Conf. Neural Netw., 2008, pp. 3479-3485.

[15] M. Pecht and R. Jaai, "A prognostics and health management roadmap for information and electronics-rich systems," Microelectron. Rel., vol. 50, no. 3, pp. 317-323, 2010.

[16] H. Oh, B. Han, P. McCluskey, Ch. Han, and B. D. Youn, "Physics-offailure, condition monitoring, and prognostics of insulated gate bipolar transistor modules: A review," IEEE Trans. Power Electron., vol. 30, no. 5, pp. 2413-2426, May 2015.

[17] R. Wu, F. Blaabjerg, H. Wang, M. Liserre, and F. Iannuzzo, "Catastrophic failure and fault-tolerant design of IGBT power electronic converters-An overview," in Proc. Annu. Conf. IEEE Ind. Electron. Soc., 2013, pp. 507-513.

[18] N. Y. A. Shammas, "Present problems of power module packaging technology," Microelectron. Rel., vol. 43, no. 4, pp. 519-527, 2003.

[19] G. Sonnenfeld, K. Goebel, and J. R. Celaya, "An agile accelerated aging, characterization and scenario simulation system for gate controlled power transistors," in Proc. IEEEAUTOTESTCON, 2008, pp. 208-215.

[20] G. Coquery, G. Lefranc, T. Licht, R. Lallemand, N. Seliger, and H. Berg, "High temperature reliability on automotive power modules verified by power cycling tests up to $150^{\circ} \mathrm{C}$," Microelectron. Rel., vol. 43, no. 9-11, pp. 1871-1876, 2003.

[21] V. Smet, F. Forest, J.-. Huselstein, F. Richardeau, Z. Khatir, S. Lefebvre, and M. Berkani, "Ageing and failure modes of IGBT modules in high-temperature power cycling," IEEE Trans. Ind. Electron., vol. 58, no. 10, pp. 4931-4941, 2011.

[22] M. Held, P. Jacob, G. Nicoletti, P. Scacco, and M.-. Poech, "Fast power cycling test of IGBT modules in traction application," in Proc. Int. Conf. Power Electron. Drive Syst., 1997, vol. 1, pp. 425-430.
[23] A. Hensler, J. Lutz, M. Thoben, and J. Zachariae, "Power cycling tests at high temperatures with IGBT power modules for hybrid electrical vehicle applications," in Proc. Electron. Syst.-Integr. Technol. Conf., 2010, pp. 1-6.

[24] N. Patil, J. Celaya, D. Das, K. Goebel, and M. Pecht, "Precursor parameter identification for insulated gate bipolar transistor (IGBT) prognostics," IEEE Trans. Rel., vol. 58, no. 2, pp. 271-276, Feb. 2009.

[25] P. Perner, Machine Learning and Data Mining in Pattern Recognition. Leipzig, Germany: Springer, 2001.

[26] A. Alghassi, S. Perinpanayagam, M. Samie, and T. Sreenuch, "Computationally efficient, real-time, and embeddable prognostic techniques for power electronics," IEEE Trans. Power Electron., vol. 30, pp. 2623-2634, May 2015.

[27] H. Qiu, J. Lee, J. Lin, and G. Yu, "Robust performance degradation assessment methods for enhanced rolling element bearing prognostics," Adv. Eng. Inf., vol. 17, pp. 127-140, 2003, 0.

[28] T. Sreenuch, A. Alghassi, S. Perinpanayagam, and Y. Xie, "Probabilistic Monte-Carlo method for modelling and prediction of electronics component life," Int. J. Adv, Comput. Sci. Applications, vol. 5, no. 1, pp. 96-104, 2014.

[29] U. Maulik and S. Bandyopadhyay, "Genetic algorithm-based clustering technique," Pattern Recogn., vol. 33, pp. 1455-1465, 2000, 9.

[30] J. Z. Sikorska, M. Hodkiewicz, and L. Ma, "Prognostic modelling options for remaining useful life estimation by industry," Mech. Syst. Signal Process., vol. 25, pp. 1803-1836, 2011, 7 .

[31] R. Derakhshani and S. A. C. Schuckers, "Continuous time delay neural networks for detection of temporal patterns in signals," in Proc. Int. Joint Conf. Neural Netw., 2004, pp. 2723-2728.

[32] H. Zhang, M. Ö. Balaban, and J. C. Principe, "Improving pattern recognition of electronic nose data with time-delay neural networks," Sensors Actuators B, Chem., vol. 96, no. 1-2, pp. 385-389, 2003.

[33] R. Sitte and J. Sitte, "Analysis of the predictive ability of time delay neural networks applied to the S\&P 500 time series," IEEE Trans. Syst. Man Cybern. C, Appl. Rev., vol. 30, no. 4, pp. 568-572, Nov. 2000.

[34] S. A. Mojarad, S. S. Dlay, W. L. Woo, and G. V. Sherbet, "Breast cancer prediction and cross validation using multilayer perceptron neural networks," in Proc. 7th IEEE IET Int. Symp. Commun. Syst. Netw. Digital Signal Process., 2010, pp. 760-764.

[35] Y. Tan, C. Su, and N. Karim, "Neural network based time-delay estimation for nonlinear dynamic systems," in Proc. IFAC 15th Triennial World Congress, Barcelona, Spain, 2002, CD-ROM.

[36] S. Rekik, S.-. Selouani, D. Guerchi, and H. Hamam, "An autoregressive time delay neural network for speech steganalysis," in Proc. 11th Int. Conf. Inf. Sci., Signal Process. and Their Applications, 2012, pp. $54-58$.

[37] G. Lera and M. Pinzolas, "Neighborhood based Levenberg-Marquardt algorithm for neural network training," IEEE Trans. Neural Netw., vol. 13, no. 5, pp. 1200-1203, Sep. 2002.

[38] Z. Tian, "An artificial neural network approach for remaining useful life prediction of equipments subject to condition monitoring," $J$. Intell. Manuf., vol. 23, pp. 227-237, 2012.

[39] L. Ma, J.-S. Kang, C.-Y. Zhao, and S.-Y. Liu, "Modeling the impact of prognostic errors on CBM effectiveness using discrete-event simulation," in Proc. Int. Conf. Quality, Rel. , Risk, Maintenance, and Safety Eng., 2012, pp. 520-525.

[40] F. O. Heimes, "Recurrent neural networks for remaining useful life estimation," in Proc. Int. Conf. Prognostics and Health Management, 2008, pp. 1-6.

[41] H. Hirose, A. Todoroki, S. Matsuda, and M. Hikita, "Electrical insulation diagnosing using a new statistical classification method," in Proc. 8th Int. Conf. Properties and Applications of Dielectric Mater., 2006, pp. 698-701.

[42] J. F. Lawless, Statistical Model and Methods for Lifetime Data, 2nd ed. Hoboken, NN, USA: Wiley, 2011

[43] R. B. Millar, Maximum Likelihood Estimation and Inference, 1st ed. Chichester, U.K.: Wiley, 2011.

[44] C. Louen, S. X. Ding, and C. Kandler, "A new framework for remaining useful life estimation using support vector machine classifier," in Proc. Control and Fault-Tolerant Syst. Conf., 2013, pp. 228-233.

[45] D. Siegel and J. Lee, "An auto-associative residual processing and K-means clustering approach for anemometer health assessment," Int. J. Prognost. Health Manag., vol. 2, pp. 50-61, 2011.

[46] Y. Xiong, X. Cheng, Z. J. Shen, C. Mi, H. Wu, and V. K. Garg, "Prognostic and warning system for power-electronic modules in electric, hybrid electric, and fuel-cell vehicles," IEEE Trans. Ind. Electron., vol. 55, no. 6, pp. 2268-2276, Jun. 2008. 
[47] B. Ji, V. Pickert, and B. Zahawi, "In-situ bond wire and solder layer health monitoring circuit for IGBT power modules," in Proc. 7th Int. Conf. Integr. Power Electron. Syst., 2012, pp. 1-6.

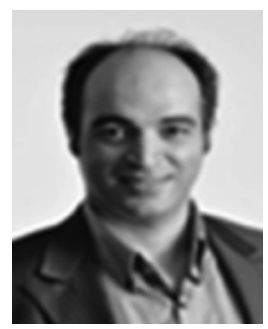

Alirezza Alghassi received the B.Sc. degree in electrical power engineering in Iran, the B.Sc. degree in control system engineering from Sheffield University, U.K., and the M.Sc. degree in power electronic engineering from Nottingham University. He is currently working toward the Ph.D. degree at the School of Aerospace, Transport and Manufacturing, Cranfield University, Bedford, U.K.

$\mathrm{He}$ is a Researcher with the Integrated Vehicle Health Management Centre, Cranfield University, Bedford, U.K.

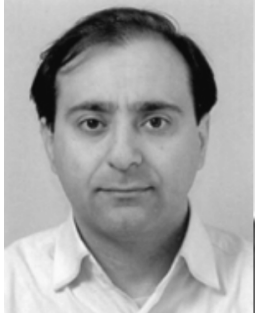

Mohammad Samie received the Ph.D. degree in advanced electronics from the University of the West of England, Bristol, U.K., in 2012.

He was involved with research on self-healing cellular architectures for biologically inspired highly reliable electronic systems funded by EPSRC, a joint research establishment between the University of the West of England and the University of Bristol. He is currently a Research Fellow with the Integrated Vehicle Health Management Centre, Cranfield University, Bedford, U.K., which is jointly funded by Boeing, Cranfield University, and a number of other industrial partners to conduct research on system health management, power electronics, and self-healing systems.

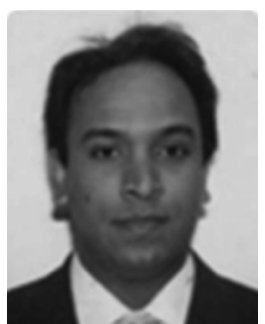

Suresh Perinpanayagam received the M.Eng. and Ph.D. degrees from Imperial College, London, U.K.

$\mathrm{He}$ is currently a Lecturer with the Integrated Vehicle Health Management Centre, Cranfield University, Bedford, U.K., where he heads the Electronics Prognistics Group, which works on highly accelerated life testing, electro-thermal modelling, thermomechanical modeling, reliability and failure assessment capabilities for renewable energy systems, automotive, aerospace and rail applications. This laboratory develops data-mining techniques for anomaly detection, diagnostics, prognostics, and predictive maintenance for power electronics from the above-mentioned industry sectors. His research group consists of one research fellow, five Ph.D. students, and seven M.Sc. students. 
2016-05-30

\section{Stochastic RUL calculation enhanced with TDNN-based IGBT failure modeling}

Alghassi, Alireza

Institute of Electrical and Electronics Engineers

Alireza Alghassi, Suresh Perinpanayagam and Mohammad Samie. Stochastic RUL calculation enhanced with TDNN-based IGBT failure modeling. IEEE Transactions on Reliability, Volume:65, Issue:2, pp558-573

https://dspace.lib.cranfield.ac.uk/handle/1826/10048

Downloaded from Cranfield Library Services E-Repository 